\title{
Caracterização química do solo das cavas de argila no Baixo Paraíba visando a restauração das áreas de reserva legal.
}

\author{
Brício Marcelino da Silva ${ }^{1}$ \\ Elaine dos Santos Pedroza ${ }^{2}$ \\ Vicente de Paulo Santos de Oliveira ${ }^{3}$ \\ ${ }^{1}$ Instituto Federal Fluminense - IFF/RJ \\ Rua Dr. Siqueira, 372 - Pq. Dom Bosco - 28030130 - Campos dos Goytacazes - \\ RJ, Brasil \\ bsilva@iff.edu.br \\ ${ }^{2}$ Instituto Federal Fluminense - IFF/RJ - Bolsista do CNPq \\ Rua Dr. Siqueira, 372 - Pq. Dom Bosco - 28030130 - Campos dos Goytacazes - \\ RJ, Brasil \\ elaine.ely@ hotmail.com \\ ${ }^{3}$ Instituto Federal Fluminense - IFF/RJ \\ Rua Dr. Siqueira, 372 - Pq. Dom Bosco - 28030130 - Campos dos Goytacazes - \\ RJ, Brasil \\ vsantos@iff.edu.br
}

\begin{abstract}
Campos dos Goytacazes city, is located on the north of Rio de Janeiro State, nowadays it is the biggest brick producer in the country. Because of that $7.000 \mathrm{~m}^{3}$ of mud is extracted daily, causing huge modifications in the environment. The main of this work is to describe the conditions of the ground of the area where the mud is extracted from. The chemical analysis of this ground showed important modifications on the chemical composition. It revealed the impact of this activity, that generally causes the lost of important components of the soil, decreasing the fertility on these environments.
\end{abstract}

Palavras-chave: cava de argila, caracterização química do solo, recuperação de área degradada.

\section{Introdução}

$\mathrm{Na}$ região norte fluminense, a indústria extrativista tem grande expressão, sendo responsável, atualmente, por $84 \%$ da produção nacional de petróleo (PETROBRAS, 2008) e na produção de cerca de 120 milhões de peças de cerâmica por mês, sendo o maior polo produtor de tijolos do país na atualidade. A Indústria sucro-alcoleira, com o cultivo extensivo de cana para produção de açúcar e álcool, também ocupa um lugar de destaque. Todas essas atividades causam grandes modificações no ambiente, seja pela extensão, como no caso das plantações de cana-de-açúcar que ocupam 200.000 hectares (PMCG, 2005, p.155), seja pelo potencial poluidor, como no caso do petróleo, ou pela destruição do solo como é o caso das cerâmicas (RAMOS, 2000).

Apesar de a indústria ceramista ser uma atividade econômica de grande importância para a região é, também, uma das que mais impactam, negativamente, o ambiente. Atualmente, as indústrias cerâmicas extraem cerca de $7.000 \mathrm{~m}^{3}$ diários de argila. Ramos (2000) estima haver no norte fluminense uma jazida total de $1.591 .460 .000 \mathrm{~m}^{3}$ de argila, com uma área total explorável de $620 \mathrm{~km}^{2}$, sendo esta, portanto, a área a ser impactada diretamente por essa atividade. Levando-se em conta as clareiras, as lavras abertas, bordaduras e aceiros tem-se uma área degradada bastante expressiva. Além da degradação do solo e da alteração da 
paisagem, esta atividade reduz a viabilidade de recuperação ambiental por deixar o solo com inúmeros aspectos restritivos ao crescimento de uma nova biota.

O solo é o substrato primordial dos ecossistemas naturais. A recuperação das funções ecológicas dessas áreas, buscando restaurar a diversidade da flora, a micro e macro fauna são, portanto, imprescindíveis (GONÇALVES et al., 2008).

Nesse intuito, conhecer as condições do solo remanescente se torna indispensável. Este trabalho visa descrever as condições do solo após a mineração de argila na região de Campos dos Goytacazes.

\section{Caracterização da região}

O município de Campos dos Goytacazes está localizado na Região Norte do Estado do Rio de Janeiro (figura 1), a $279 \mathrm{~km}$ da capital estadual, sendo o maior município do Estado com uma área de, aproximadamente, $4.037 \mathrm{~km}^{2}$ (IBGE, 2008).

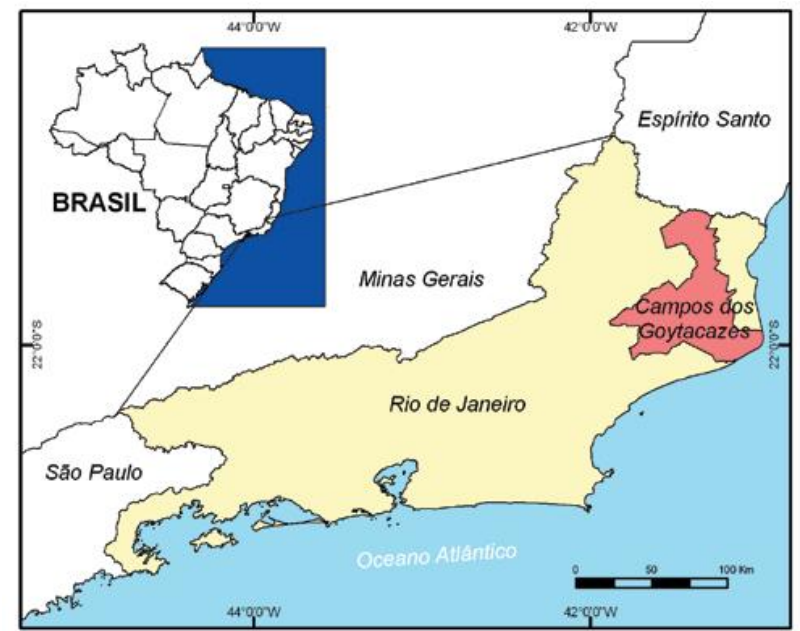

Figura 1 - Mapa de localização do município de Campos dos Goytacazes (IBGE, 2008).

O clima do município é quente e úmido com estação chuvosa no verão e estiagem no inverno. As precipitações pluviométricas médias anuais variam entre 1.100 e $1.300 \mathrm{~mm}$. A maior ocorrência de chuvas ocorre nos meses de janeiro e fevereiro. A temperatura média anual varia entre 19 a $23^{\circ} \mathrm{C}$, com máxima de $34^{\circ} \mathrm{C}$ (SIPRJ, 2008).

Os sedimentos encontrados nesta região são constituídos por argilas e siltes, micáceos, de coloração acinzentada, e areias quartzosas de coloração branco-amarelada. A argila é um sedimento característico de coloração cinza-negra e textura plástica. Pode estar associada a depósitos de diatomita ou, quando em pântanos e brejos, associada à turfa. (PROJIR, 1984).

Na região em estudo, as áreas onde são encontradas as jazidas de argila estão à margem direita do Rio Paraíba do Sul, ao longo da estrada RJ-216 que liga Campos dos Goytacazes ao Farol de São Tomé, estando a sua maior concentração próxima aos vilarejos de São Sebastião, Poço Gordo e do Mosteiro de São Bento. Esses depósitos são típicos de planície de inundação, estando ligados à flutuação do curso do rio, desde a Lagoa Feia até a foz atual (RAMOS, 2000).

A caracterização das argilas provenientes dessas diferentes áreas de extração classificamna como inorgânica, de elevada plasticidade, com teor de matéria orgânica inferior a $1 \%$, com predominância de caulinita, sendo notada a presença de quartzo, mica, anfibolitos, zircão, gibsita, goetita e codierita, todas dentro dos padrões obtidos por outras argilas nacionais. (ALEXANDRE, 2000). 


\section{Características da lavra}

O levantamento das características do processo de extração de argila, nas áreas mineradas, foi obtido diretamente nas cavas, em entrevistas não estruturadas com os proprietários, funcionários que trabalhavam na extração ou com integrantes do Sindicato dos Ceramistas de Campos dos Goytacazes.

Foram escolhidas para a amostragem dez cavas distribuídas espacialmente na jazida de modo a se ter uma amostra representativa do todo. Estão nas localidades de: Barcelos, Saquarema Grande, Goytacazes, Poço Gordo, Campo Limpo, Tocos, Mussurepe, Santo Amaro e Pipeiras.

A exploração da jazida de argila em Campos dos Goytacazes é feita da seguinte forma: primeiro é retirado, por raspagem, o horizonte A, que é um horizonte mineral com acúmulo de matéria orgânica misturada com o material mineral da superfície ou adjacente a ela (Ramos, 2000), com o propósito de expor o pacote argiloso. Em seguida, é iniciada a extração da argila, utilizando-se retro-escavadeiras (Figura 2).

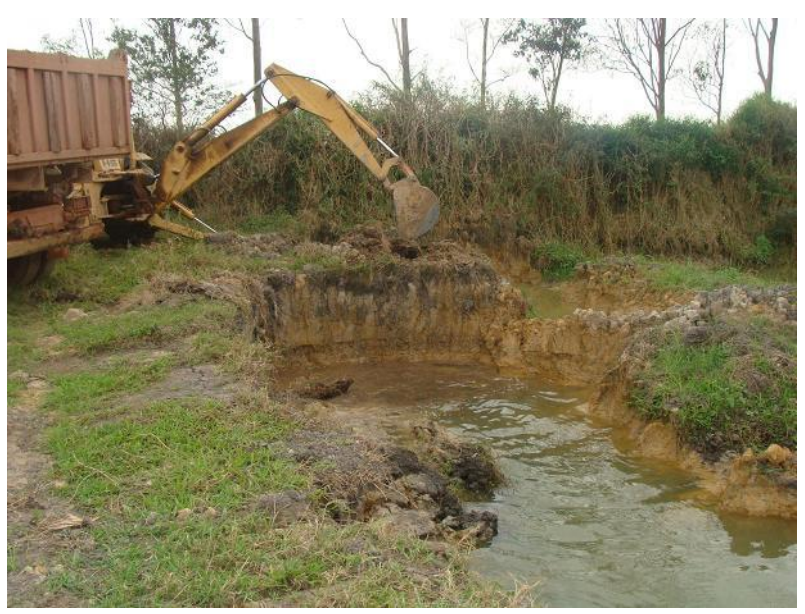

Figura 2 - Extração de argila (Foto: do autor)

Valicheski (2004) salienta que, na maioria dos casos, a extração de argila é realizada sem critérios pré-estabelecidos objetivando a reutilização da área, dificultando o processo de revegetação e recuperação ambiental dessas áreas, uma vez que o substrato remanescente apresenta características químicas pouco propícias para o desenvolvimento da vegetação e da fauna.

Este autor aponta em seu estudo que a maior parte das áreas exploradas encontram-se abandonadas, estando de acordo com o que observamos. As possíveis causas para essa realidade é que grande parte das jazidas (arrendadas) não possuem licença ambiental, não sendo assumido pelo minerador, o compromisso de recuperação. Outra causa provável é que, como o processo de extração é realizado de modo empírico, sem visar à reutilização da área após a exploração, resultando na baixa qualidade do substrato remanescente, isso dificulta ou mesmo impossibilita a sua reutilização para outros fins (VALICHESKI, 2004).

Das dez cavas pesquisadas, quatro tiveram o horizonte A do solo incorporado à argila e enviado para a olaria (figura 3), apesar de essa prática diminuir a qualidade do produto final da olaria e privar o solo de sua porção de matéria orgânica superficial.

Essa retirada acarreta a perda parcial ou total da capacidade de sustentar o crescimento de plantas e outros organismos, ocorrendo a degradação dos atributos físicos, químicos e biológicos do solo, altamente interdependentes, comprometendo a qualidade do mesmo (GONÇALVES et al, 2008). 


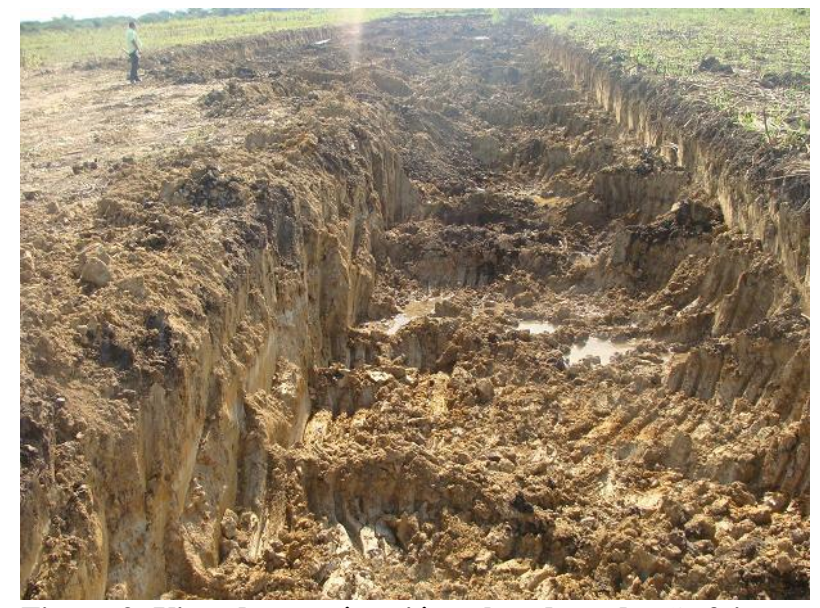

Figura 3: Vista de uma área já explorada onde não foi depositado o horizonte A do solo (Foto: do autor).

Em alguns casos, como na localidade de Mussurepe, o lençol freático é tão próximo da superfície que, quando acontece a extração do pacote argiloso, imediatamente a cava é preenchida com água impossibilitando a implantação de qualquer atividade de recuperação do solo.

\section{Atributos biológicos, físicos e químicos do solo}

Para avaliar a qualidade do solo pode-se realizar o monitoramento de seus atributos ou características biológicas, físicas e químicas (CARVALHO et al. 2004). Outras definições para qualidade do solo são encontradas nos trabalhos de Manzatto (1998), Silva (2009), Larson e Pierce (1991), Johnson et al. (1997) e outros.

Os atributos biológicos são mantidos por uma vasta e diversa comunidade de organismos que mantém entre si complexas relações e influenciam as propriedades físicas, químicas e biológicas do solo (ASSAD, 1997 apud SILVA, 2009). Suas atividades contribuem para a ciclagem de nutrientes, mantém baixas as populações de pragas, produzem substâncias que promovem a formação de agregados do solo e produzem substâncias húmicas, sendo a maioria dos organismos muito importantes para a qualidade do solo (MAGDOFF, 2002).

A diversidade da fauna do solo tem sido considerada um importante indicador biológico, sensível às alterações ecológicas nos ecossistemas, pois contribui na manutenção da estrutura e fertilidade dos solos tropicais (BROWN et al., 2003).

Quanto aos atributos físicos, no que diz respeito à funcionalidade, a estrutura do solo ideal é aquela que permite uma grande área de contato entre as raízes e o solo, apresentando espaço poroso suficiente para a movimentação de água e difusão de gases, além da resistência à penetração não ser impeditiva ao crescimento das raízes (VALICHESKI, 2004).

Esse autor especifica como qualidade física do solo, a capacidade de ele promover ao sistema radicular das plantas as condições favoráveis ao crescimento e desenvolvimento e é determinada pela disponibilidade de água, aeração, temperatura e resistência à penetração (VALISHESKI, 2008).

Outro aspecto importante a ser considerado está relacionado com a proximidade do lençol freático, que, devido ao excesso de umidade, dificulta o desenvolvimento do sistema radicular da maioria das espécies arbóreas, devido à deficiente oxigenação do solo nessas condições (ANDRADE, 1997) ou ainda possibilita a formação de solos salinos, pois intensifica a ascensão capilar levando a um acúmulo gradual de sais na superfície. Como a baixada campista possui elevada quantidade de sais solúveis no lençol freático, esse risco torna-se muito maior (IAA/MIC, 1984). 
Carvalho salienta outros atributos físicos ou indicadores, tais como densidade e porosidade, estado de agregação e de compactação. (CARVALHO et al., 2004)

Os nutrientes disponíveis, $\mathrm{C}$ orgânico total, e $\mathrm{pH}$ foram os atributos químicos avaliados. Foi quantificada, também, a matéria orgânica no solo, compreendendo aí, raízes de plantas, a fauna, microrganismos do solo, substâncias humificadas e não humificadas.

\section{Avaliação do solo remanescente}

A tabela 1 apresenta o resultado das análises químicas completa dos solos encontrados nas cavas selecionadas, a partir de amostras compostas, obtidos pela mistura do material de solo coletado em diversos pontos do terreno. Comparamos os resultados com Almeida et al. (1988) que apresenta classificação para análise de níveis fósforo $(\mathrm{P})$, potássio $(\mathrm{K})$, alumínio $(\mathrm{Al})$, cálcio mais magnésio $(\mathrm{Ca}+\mathrm{Mg})$, potencial de hidrogênio $(\mathrm{pH})$ e carbono $(\mathrm{C})$ para o estado do Rio de Janeiro.

Tabela 1. Propriedades químicas de substrato da cava de extração de argila na camada de $0,20 \mathrm{~m}$ de profundidade.

\begin{tabular}{|c|c|c|c|c|c|c|c|c|c|c|c|}
\hline & $\mathrm{pH}$ & $\mathrm{P}$ & $\mathrm{K}$ & $\mathrm{Ca}$ & $\mathrm{Mg}$ & $\mathrm{Al}$ & $\mathrm{H}+\mathrm{Al}$ & $\mathrm{Na}$ & $\mathrm{C}$ & $\mathrm{MO}$ \\
\hline & \multicolumn{2}{|c|}{$\mathrm{mg} / \mathrm{dm}^{3}$} & \multicolumn{7}{|c|}{$\mathrm{cmol}_{\mathrm{c}} / \mathrm{dm}^{3}$} & $\%$ & $\mathrm{~g} / \mathrm{dm}^{3}$ \\
\hline Cava 1 & 5,9 & 10 & 32 & 3,6 & 2,6 & 0,1 & 3,4 & 0,45 & 0,87 & 15,0 \\
\hline Cava 2 & 5,7 & 19 & 60 & 3,8 & 2,6 & 0,2 & 4,2 & 0,31 & 0,91 & 15,7 \\
\hline Cava 3 & 7,4 & 10 & 25 & 7,6 & 3,1 & 0,0 & 0,8 & 2,09 & 0,67 & 11,6 \\
\hline Cava 4 & 7,1 & 27 & 29 & 4,0 & 2,5 & 0,0 & 2,2 & 0,60 & 0,55 & 9,5 \\
\hline Cava 5 & 6,3 & 10 & 8 & 7,2 & 4,3 & 0,0 & 1,1 & 4,70 & 0,44 & 7,6 \\
\hline Cava 6 & 6,7 & 11 & 30 & 3,7 & 2,1 & 0,0 & 1,7 & 2,74 & 0,79 & 13,6 \\
\hline Cava 7 & 5,7 & 27 & 49 & 9,1 & 5,1 & 0,0 & 3,3 & 1,67 & 1,36 & 23,4 \\
\hline Cava 8 & 6,7 & 11 & 19 & 2,9 & 3,3 & 0,0 & 2,3 & 0,90 & 0,59 & 10,2 \\
\hline Cava 9 & 5,9 & 14 & 82 & 1,3 & 1,4 & 0,2 & 2,5 & 0,96 & 0,48 & 8,3 \\
\hline Cava 10 & 7,4 & 114 & 40 & 4,0 & 0,6 & 0,0 & 0,7 & 0,15 & 0,95 & 16,4 \\
\hline
\end{tabular}

Tabela 1 (continuação). Propriedades químicas de substrato da cava de extração de argila na camada de $0,20 \mathrm{~m}$ de profundidade.

\begin{tabular}{|c|c|c|c|c|c|c|c|c|c|}
\hline & SB & $\mathrm{T}$ & $\mathrm{t}$ & $\mathrm{m}$ & $\mathrm{V}$ & $\mathrm{Fe}$ & $\mathrm{Cu}$ & $\mathrm{Zn}$ & $\mathrm{Mn}$ \\
\hline & \multicolumn{3}{|c|}{$\mathrm{cmol}_{\mathrm{c}} / \mathrm{dm}^{3}$} & \multicolumn{3}{c|}{$\%$} & \multicolumn{5}{c|}{$\mathrm{mg} / \mathrm{dm}^{3}$} \\
\hline Cava 1 & 6,7 & 10,1 & 6,8 & 1 & 66 & 244,2 & 4,0 & 4,5 & 76,8 \\
\hline Cava 2 & 6,9 & 11,1 & 7,1 & 3 & 62 & 545,6 & 7,7 & 5,9 & 146,4 \\
\hline Cava 3 & 12,9 & 13,7 & 12,9 & 0 & 94 & 72,0 & 4,4 & 3,2 & 21,3 \\
\hline Cava 4 & 7,2 & 9,4 & 7,2 & 0 & 77 & 99,0 & 2,4 & 4,0 & 29,5 \\
\hline Cava 5 & 16,2 & 17,3 & 16,2 & 0 & 94 & 77,4 & 1,8 & 2,4 & 23,6 \\
\hline Cava 6 & 8,6 & 10,3 & 8,6 & 0 & 84 & 238,2 & 6,4 & 3,6 & 84,0 \\
\hline Cava 7 & 16,0 & 19,3 & 16,0 & 0 & 83 & 131,4 & 10,8 & 24,6 & 68,4 \\
\hline Cava 8 & 7,1 & 9,4 & 7,1 & 0 & 76 & 88,8 & 3,3 & 3,8 & 23,3 \\
\hline Cava 9 & 3,9 & 6,4 & 4,1 & 5 & 61 & 145,8 & 4,7 & 2,6 & 12,9 \\
\hline Cava 10 & 4,9 & 5,6 & 4,9 & 0 & 87 & 142,8 & 19,2 & 6,0 & 45,6 \\
\hline
\end{tabular}


$\mathrm{O} \mathrm{pH}$ variou de moderadamente ácido a moderadamente alcalino $(5,7$ a 7,4), sendo que na maioria das análises classificou-se como moderadamente ácido.

Os níveis de fósforo encontrados em $70 \%$ das amostras ficaram na classe de fertilidade muito baixa (menor de $14 \mathrm{mg} / \mathrm{dm}^{3}$ ) e o restante como baixa (entre 14 e $28 \mathrm{mg} / \mathrm{dm}^{3}$ ). O mesmo comportamento foi observado para o potássio $(\mathrm{K})$ onde também na maioria das cavas $(70 \%)$ ficou na classe de fertilidade muito baixa e baixa (30\%).

Considerando os níveis de cálcio e magnésio encontrados nas análises, as classes de fertilidade foram classificadas de médio a muito alto, sendo que na maioria das cavas predominaram as classes alto e muito alto. $\mathrm{O}$ alumínio não foi detectado na maioria das análises $(70 \%)$ e quando ocorreu se deu em baixos níveis.

Considerando recomendações a fim de elevar os níveis de cálcio $(\mathrm{Ca})$ e magnésio $(\mathrm{Mg})$ encontrados e neutralizar a ação do alumínio para espécies florestais (ALMEIDA et al., 1988), conclui-se que nas cavas estudadas não será necessária a realização de calagem. Os valores encontrados de Soma de Bases (S.B.), Capacidade de Troca Catiônica (T e t), Saturação de Alumínio (m) e Saturação de Base (V) confirmam este resultado.

As altas saturações por bases (valor $\mathrm{V}>60 \%$ ) e os elevados teores de bases trocáveis dos perfis estudados pode ser conseqüência de elevados teores de cálcio e magnésio. O sódio, quando apresenta elevada concentração, também tem grande influência no valor $\mathrm{V}$. Nas condições das cavas, dependendo da capacidade de retenção de cátions, o teor deste elemento químico pode alcançar nos solos níveis tóxicos e intolerantes para as culturas (VALISHESKI, 2008).

Em $90 \%$ das amostras analisadas os níveis de carbono (C) foram classificados como baixo nível de fertilidade.

No que diz respeito ao suprimento de fósforo $(\mathrm{P})$, para espécies florestais não será necessário adubação, por outro lado, será preciso o equivalente a $30 \mathrm{~kg} / \mathrm{ha}$ de $\mathrm{K}_{2} 0$ (óxido de potássio) em adubo mineral ou orgânico.

O teor total médio do cobre $(\mathrm{Cu})$ nos solos em geral varia de 10 a $80 \mathrm{mg} / \mathrm{kg}$. Os valores estimados nas análises variam de 1,8 e $10,8 \mathrm{mg} / \mathrm{dm}^{3}$, podendo em todos os casos ser considerados baixos. O cobre é constituinte presente em certas enzimas, além de influenciar na fixação do nitrogênio atmosférico pelas leguminosas e é essencial no balanço de nutrientes que regulam a transpiração das plantas (NOVAIS et al., 2007).

Os níveis de ferro (Fe) nos solos das cavas variaram de 72 a $545,6 \mathrm{mg} / \mathrm{dm}^{3}$, enquanto, segundo NOVAIS et al. (2007), a concentração média encontrada em solo é de $38 \mathrm{mg} / \mathrm{kg}$. Este elemento opera na ativação de enzimas, atuando na catalisação da biossíntese da clorofila, já que faz parte constituinte de enzimas responsáveis pela sua formação.

O manganês (Mn) no solo é componente de óxidos, carbonatos, silicatos e sulfetos. Os resultados das análises estimaram valores variando entre 12,9 e $146,4 \mathrm{mg} / \mathrm{dm}^{3}$, níveis baixos comparados aos teores médios encontrados nos solos (faixa entre 20 a $3000 \mathrm{mg} / \mathrm{kg}$ ). É necessário à síntese de clorofila, sendo que sua função principal está relacionada com a ativação de enzimas.

O teor de zinco $(\mathrm{Zn})$ variou nos resultados entre 2,4 a $24,6 \mathrm{mg} / \mathrm{dm}^{3}$ nas análises dos solos nas cavas. Este micronutriente atua como cofator enzimático, sendo essencial para a atividade, regulação e estabilização da estrutura protéica ou uma combinação destas. Nos solos os seus teores variam geralmente encontram-se na faixa de 10 a $300 \mathrm{mg} / \mathrm{kg}$, se apresentando mais disponível em solos com $\mathrm{pH}$ ácido.

Considerado como um elemento mineral benéfico por estimular o crescimento das plantas (NOVAIS et al., 2007), o sódio (Na) foi encontrado nas análises em teores variando entre 0,60 a $4,70 \mathrm{cmol}_{\mathrm{c}} / \mathrm{dm}^{3}$. 
Porém, um elevado teor de sódio no complexo sortivo do solo, com altas concentrações de sódio trocável, resulta em severas restrições as atividades agrícolas. Valisheski (2008) classifica os solos remanescentes da atividade de extração de argila, em todas as suas pesquisas na região em questão, em severamente afetados pelo sódio, apresentando caráter sódico.

A matéria orgânica desempenha grande influência sobre propriedades físicas, químicas e biológicas, tendo uma função primordial na qualidade e no uso sustentável do solo. Seus teores nas cavas variaram entre 8,3 a $23,4 \mathrm{~g} / \mathrm{dm}^{3}$. A operação de retirada de argila nas cavas geralmente promove a remoção deste importante componente, reduzindo a sua fertilidade.

\section{Conclusão}

A partir das análises completas de solo realizadas em 10 cavas de extração de argila na Baixada Campista pode-se constatar que o $\mathrm{pH}$ foi classificado como moderadamente ácido na maioria das amostras, os teores de fósforo $(\mathrm{P})$ e potássio $(\mathrm{K})$ situaram entre muito baixo a baixo, as bases do solo ( $\mathrm{Ca}, \mathrm{Mg}$ e $\mathrm{K}$ ) estão presentes a níveis médio a muito alto, não havendo necessidade de calagem. O nível de carbono encontrado (C) o classifica como solo com baixo nível de fertilidade, no entanto, foi registrada a presença de micronutrientes importantes no desenvolvimento das espécies florestais ( $\mathrm{Fe}, \mathrm{Mn}$ e $\mathrm{Zn}$ ). Os teores de matéria orgânica (MO) variaram de 8,3 a $23,4 \mathrm{~g} / \mathrm{dm}^{3}$, confirmando que a operação de retirada de argila nas cavas geralmente promove a remoção deste importante componente, reduzindo a fertilidade dos solos nestes ambientes. As concentrações de sódio devem ser consideradas, pois, nas condições encontradas, esse elemento químico pode apresentar efeito fitotóxico para várias espécies vegetais, que resulta em severas restrições para a reutilização dessas áreas para fins agrícolas após a extração da argila.

\section{REFERÊNCIAS BIBLIOGRÁFICAS}

Alexandre, J. Análise de matérias-primas e composição de massa utilizada em cerâmicas vermelhas. 2000, 174p. Tese (Doutorado em ciência de Engenharia) - Universidade Estadual do Norte Fluminense - UENF, Campos dos Goytacazes. RJ. 2000.

Almeida, D. L.; Santos, G. A.; De-Polli, H.; Cunha, L. H.; Freire, L. R.; Amaral Sobrianho, N. M. B.; Pereira, N. N. C.; Eira, P. A.; Bloise, R. M.; Salek, R. C. Manual de adubação para o estado do Rio de Janeiro. Coleção Universidade Rural - Série Ciências Agrárias No 2. Seropédica, RJ, Brasil. 179p. Editora Universidade Rural, 1988.

Andrade, A.G.; Costa, G.S. \& Faria, S.M. Decomposição e deposição da serapilheira em povoamentos de Mimosa caesalpiniifolia, Acacia mangium e Acacia holosericea com quatro anos de idade em Planossolo. Revista Brasileira de Ciência do Solo, 24:777-785, 2000.

Anjos, L.H.C. Caracterização, gênese, classificação e aptidão agrícola de uma sequencia de solos do terciário na região de Campos-RJ. Dissertação. Universidade Federal Rural do Rio de Janeiro - UFRRJ, Itaguaí, 194p. 1985.

Brown, G.G.; Benito, N.P.; Pasini, A.; Sautter, K.D.; Guimarães, M.F.; Torres, E. No-tillage greatly increases earthworm populations in Paraná state, Brazil. Pedobiologia, Jena, v.47, p.764-771, 2003.

Campos dos Goytacazes, Prefeitura Municipal. Campos dos Goytacazes: Perfil 2005. Campos dos Goytacazes: Prefeitura Municipal de Campos dos Goytacazes, Instituto Superior de Ensino do CENSA, FUNDENOR, 2006.

Carvalho, R. Goedert W.J. Armando, M.S. Atributos físicos da qualidade de um solo sob sistema agroflorestal. Pesquisa agropecuária brasileira, Brasília, v.39, n.11, p.1153-1155, nov. 2004

Gonçalves, J.L.M. O sistema radicular da árvore. in: Gonçalves, J.L.M. Benedetti, V V. Nutrição e fertilização florestal. Piracicaba: IPEF, 219-269P. 2008 
IAA/MIC. Instituto do Açúcar e do Álcool / Ministério da Indústria e Comércio. Projeto de irrigação e drenagem da cana-de-açúcar na região Norte Fluminense. Projeto de irrigação e drenagem da cana-deaçúcar na Região Norte-Fluminense; relatório técnico setorial. (Vários volumes). 1983.

Instituto Brasileiro de Geografia e Estatística (IBGE). Projeto RADAMBRASIL Levantamento de recursos naturais, Folhas SE 24 - Rio Doce. Rio de Janeiro: Ministério de Minas e Energia. 584p. 1987

Johnson, D.L.; Ambrose, S.H.; Basset, J.J.; Bowen, M.L. Crummey, D.E.; Isaacson, J.S.; Johnson, D.N.; Lamb, P.; Saul, M.; Winter-Nelson, A.E. Meanings of environmental terms. Journal of Environmental Quality, New York, v.26, p.581-589, 1997.

Lamego, A. R. O Homem e o Brejo. Rio de Janeiro: Ministério da Agricultura, Serviço Gráfico do Instituto Brasileiro de Geografia e Estatística. 1945.

Larson, W.E. \& Pierce, F.J. Conservation and enhancement of soil quality. In: International Board for Soil Research and Management, 12., Bangkok, 1991. V.2.

Manzatto, C.V. Pedogênese topossequencial de solos desenvolvidos de sedimentos do terciário do Norte Fluminense: Um subsídio ao manejo agrícola racional, 1998. 148f. Tese (Doutorado em Produção Vegetal) - Universidade Estadual do Norte Fluminense, Campos dos Goytacazes, 1998.

Novais, R. F.; Alvarez V., V. H.; Barros, N. F.; Fontes, R. L. F.; Cantarutti, R. B.; Neves, J. C. L. Fertilidade do solo. Sociedade Brasileira de Ciência de Solo. Viçosa, MG. 1017p. 2007.

Petrobrás. Bacia de Campos - A maior reserva de petróleo do Brasil. Disponível em: http:// www2.petrobras.com.br/Petrobras/portugues/plataforma/pla_bacia_campos.htm Acessado em: 27/10/08.

Projeto de irrigação e drenagem da cana-de-açúcar na região Norte-fluminense (PROJIR). Ministério da Indústria e do Comércio, Instituto do Açúcar e do Álcool, Sondotécnica - Engenharia de Solos S.A. Projeto de irrigação e drenagem da cana-de-açúcar na região Norte-Fluminense: Relatórios Técnicos Setoriais; Estudos e Levantamentos Pedológicos. Campos dos Goytacazes, 1984.

Ramos, I. S. Delimitação, caracterização e cubagem da região de exploração de argila no Município de Campos dos Goytacazes. Dissertação (Mestrado em ciências de Engenharia) - Campos dos Goytacazes RJ, Universidade Estadual do Norte Fluminense - UENF, 2000.

Silva, C.F. Atributos químicos e biológicos do solo em cavas de extração de argila revegetadas com eucalipto e leguminosas. 2009. 159 f.:il. Tese (Doutorado em Produção Vegetal) - Universidade Estadual do Norte Fluminense, Campos dos Goytacazes, 2009

Valicheski, R.R. Avaliação técnica e econômica da reutilização de áreas de extração de argila em campos dos goytacazes - RJ. 2004. 132 f. Dissertação (Mestrado em Produção Vegetal) - Campos dos Goytacazes - RJ, Universidade Estadual do Norte Fluminense - UENF, 2004.

Valicheski, R.R. Atributos químicos do solo, estado nutricional e desenvolvimento radicular do coqueiro anão verde cultivado em duas classes de solos sob diferentes níveis de compactação e umidade. 2008, 99 f. il. Tese (Doutorado em Produção Vegetal) - Campos dos Goytacazes - RJ, Universidade Estadual do Norte Fluminense - UENF, 2008

\section{Sites Oficiais:}

Instituto Brasileiro de Geografia e Estatística (IBGE). Disponível em: 〈http: // www.ibge.gov.br〉, data de acesso: 02 de fevereiro de 2009.

Sistema de Informação Pluviométrica do Rio de Janeiro (SIPRJ). Disponível em http://www.cbmerj.rj.gov.br/simerj/clima_estatistica/menu_estatisticas.htm, data de acesso, 02 de fevereiro de 2009.

Agronomia.net. Principais características de adubos nitrogenados. Disponível em http://www.agronomianet.com.br/apêndice.htm, data de acesso, 20 de setembro de 2009. 